\title{
Conic Reconstruction and Correspondence from Two Views
}

\author{
Long QUAN
}

\begin{abstract}
Conics are widely accepted as one of the most fundamental image features together with points and line segments. The problem of space reconstruction and correspondence of two conics from two views is addressed in this paper. It is shown that there are two independent polynomial conditions on the corresponding pair of conics across two views, given the relative orientation of the two views. These two correspondence conditions are derived algebraically and one of them is shown to be fundamental in establishing the correspondences of conics. A unified closed-form solution is also developed for both projective reconstruction of conics in space from two views for uncalibrated cameras and metric reconstruction from calibrated cameras.

Experiments are conducted to demonstrate the discriminality of the correspondence conditions and the accuracy and stability of the reconstruction both for simulated and real images.
\end{abstract}

Keywords - conic, stereo correspondence, reconstruction.

\section{INTRODUCTION}

In computer vision, conics are widely accepted as one of the most fundamental image features together with points and straight lines. Conics are more compact and more global features than points and lines. Conics are invariant, as are points and lines under projective transformations. In addition, the mathematical properties of conics or general quadric surfaces have been thoroughly studied within algebraic projective geometry, which provides strong mathematical support. Moreover, unlike points and lines, conics contain sufficient information to impose correspondence conditions, which is very attractive for applications. Several authors have remarked the importance of conics as basic image features and developed procedures for pose estimation, stereo and motion based on conics, for instance [1], [2], [3], [4], [5], [6], [7], [8]. However, there are fewer articles dealing with conics than those devoted to

Long Quan is with LIFIA-CNRS-INRIA, 46, avenue Felix Viallet, 38031 Grenoble, France. E-mail: Long.Quan@imag.fr. points and lines.

In this paper, we are interested in the problem of conic correspondences and that of reconstruction of conics in space from two views. The importance of these issues is no doubt evident within the classical approaches of stereo vision and shape from motion. Another important motivation for this study arises from the study of invariants of conics in space [9], [10], where projective reconstruction of conics from uncalibrated cameras is essential.

We propose to solve the problems of conic correspondence and conic reconstruction from two views within a unified framework in this paper for both calibrated and uncalibrated cameras. The way we proceed is first to reformulate the problem using projective geometry based on the projective properties of quadric surfaces. Then, linear algebra is used to analyze the eigen system of a matrix pencil of order four coming from the pencil of quadric surfaces. This analysis in terms of projective geometry reveals the essential properties of conic reconstruction from a pair of images. It turns out a very simple closed form solution for reconstruction and discriminant polynomial correspondence conditions.

The original contributions of this paper are twofold:

- It is established that there are two independent polynomial conditions which should be satisfied for a pair of corresponding conics. These two a priori conditions are algebraically independent. It is then demonstrated that one of the two conditions is more important and can be used in practice for establishing conic correspondences. An invariant interpretation of this fundamental condition is also provided.

- A unified simple closed form solution for both projective and Euclidean reconstruction of conics in space is developed. The reconstruction procedure is essentially linear in that the two solutions of reconstruction are solved together with only linear computation. Only the 
extraction of the two different solutions may need to solve a quadratic equation. It is also clarified that the solutions to conic reconstruction are generally ambiguous up to two solutions and are unique only for non-transparent objects.

This work is closely related to those of $\mathrm{Ma}$ et. al [11], [3] and Safaee-Rad tt. al [4]. They both worked on the conic reconstruction problem with only fully calibrated pair of views and proposed different solutions to the problem.

Ma et al. in [11]. [3] developed an analytical method which reconstructs directly the position and orientation of the conic in space, and proposed a criterion for correspondence verification. The approach was developed and mostly limited to Euclidean framework, hence some properties regarding projective quadrics cannot be exploited in this framework. The matching criterion is more of an a posteriori verification procedure, mixed up with the reconstruction procedure.

In [4], Safaee-Rad et al. observed the projective property of a pencil of quadrics, then proposed a procedure to reconstruct the plane on which the conic in space lies. However, the solution proposed by Safaee-Rad $e t$ al. requires that a high (fourth) degree polynomial equation be solved. This is due primarily to lack of further investigation of the problem. The important properties related to the special pencil of matrices are not exploited. The independency of derived equations was not analyzed, therefore the correspondence conditions could not be made explicit. The uniqueness issue of reconstruction was also discussed in [4].

The remaining sections are organized as follows. In Section II, some preliminaries concerning the camera model and the description of two views are provided. Then the problem is formulated in Section III. Section IV gives the two polynomial correspondence conditions and some discussion on their algebraic implications. Next, Section $V$ gives the analytical method which allows one to extract two solutions in closed form. Experimental results are presented in Section VII. Finally, some concluding comments are given in Section VIII.

\section{Preliminaries}

Some basic concepts related to camera models and geometric/algebraic description of two views are briefly presented in this Section. For more details, one can refer to [12], [13], [14].

\section{A. Projection matrix of a camera}

If we assume a perspective projection for camera model, then the object space $\mathcal{R}^{3}$ may be considered as embedded in $\mathcal{P}^{3}$ (projective space of dimension 3 ) and the image space $\mathcal{R}^{2}$ embedded in $\mathcal{P}^{2}$. The camera performs the projection between $\mathcal{P}^{3}$ and $\mathcal{P}^{2}$, and this projection is represented by a $3 \times 4$ matrix $\mathbf{P}$ of rank 3 whose kernel is the projection center, $\mathbf{P}$ being called the projection matrix of the camera. The relationship between an image point in its homogeneous coordinates $\mathbf{u}=(u, v, w)^{T}$ in $\mathcal{P}^{2}$ and a space point in its homogeneous coordinates $\mathbf{x}=(x, y, z, t)^{T}$ in $\mathcal{P}^{3}$ are linearly related by $\lambda \mathbf{u}^{T}=\mathbf{P x}$, where $\lambda$ is any nonzero scalar.

The projection matrix may be decomposed into the following form

$$
\mathbf{P}=\mathbf{A}\left(\mathbf{I}_{3} \mathbf{0}_{3}\right)\left(\begin{array}{cc}
\mathbf{R} & \mathbf{t} \\
\mathbf{0}_{3}^{T} & 1
\end{array}\right),
$$

where $\mathbf{A}$, a $3 \times 3$ upper triangular matrix, accounts for the five intrinsic parameters of the camera, and $\mathbf{R}$ a space rotation matrix, together with $\mathbf{t}$ a space translation vector, account for the six extrinsic parameters.

\section{B. Realization of projection matrices for two views}

When we are dealing with two views taken at different placements of the camera(s), each view should be associated with a projection matrix which is consistent with the other one. This realization of projection matrices may be different according to the knowledge that we have on the intrinsic/extrinsic parameters of the cameras and the relative orientation of the two views. In what follows, we will examine some frequent cases.

\section{B.1 Two views from calibration}

If the two views are taken by a fully off-line calibrated stereo system (using calibration objects), we have the most complete description of the two views through the two projection matrices $\mathbf{P}$ and $\mathbf{P}^{\prime}$, each of which has the same form as (1) being, directly issued from the calibration process. The resulting $3 D$ reconstruction will be fully metric, defined in the world coordinate system which was fixed during the calibration step. 


\section{B.2 Two views from motion}

If the two views are taken by a moving calibrated camera. The motion of the camera is determined only up to the length of the translation vector. This is also equivalent to knowing the essential matrix $\mathbf{E}$ ( $c f .[15])$ of the two views. In this case, the choice of a particular world coordinate system is arbitrary, the projection matrices can therefore be taken for two views as

$\mathbf{P}=\mathbf{A}\left(\mathbf{I}_{3} \mathbf{0}_{3}\right)$ and $\mathbf{P}^{\prime}=\mathbf{A}^{\prime}\left(\mathbf{I}_{3} \mathbf{0}_{3}\right)\left(\begin{array}{cc}\mathbf{R} & \mathbf{t} /\|\mathbf{t}\| \\ \mathbf{0}_{3}^{T} & 1\end{array}\right)$.

The motion components $\mathbf{R}$ and $\mathbf{t}$ can also be obtained by factorizing $\mathbf{E}$ as suggested in [16].

The resulting $3 D$ reconstruction with this realization of rojection matrices will be Euclidean up to a global scaling factor, defined up to a rigid transformation of the placement of the first camera.

\section{B.3 Two views from epipolar geometry}

If the two views are taken by an uncalibrated moving camera, only the epipolar geometry between the two views was estimated. This is also called weakly calibrated views in [17]. The epipolar geometry can be nicely coded by a $3 \times 3$ rank 2 matrix F, called fundamental matrix [18], [19], [17]. According to Hartley [13], given a decomposition of $\mathbf{F}$ such that $\mathbf{F}=[\mathbf{t}]_{\times} \mathbf{M}$, where $\mathbf{M}$ is a non singular $3 \times 3$ matrix, $[\mathbf{t}]_{\times}$is the antisymmetric $3 \times 3$ matrix associated to the kernel vector $\mathbf{t}$ of $\mathbf{F}$, one possible choice of projection matrices for two views consistent with the above decomposition of F might be

$$
\mathbf{P}=\left(\begin{array}{ll}
\mathbf{I}_{3} & \mathbf{0}_{3}
\end{array}\right) \text { and } \quad \mathbf{P}^{\prime}=(\mathbf{M ~ t})
$$

In this case, all quantities are purely of projective nature, the resulting $3 D$ reconstruction could be no more metric, it will only be projective, defined up to a projective transformation of the placement of the first camera.

\section{B.4 Summary}

In summary, the key point is that whereever we are given two views, we obtain two consistent projection matrices $\mathbf{P}$ and $\mathbf{P}^{\prime}$ for the two views. This will allow us to reformulate conic reconstruction and correspondence from two views taken either by calibrated or uncalibrated cameras in a unified framework.

It should also be stressed that the metric information contained in each realization of projection matrices is different, from the least metric description with merely fundamental matrix, to the most metric one with fully calibrated cameras. This difference of the projection matrix realization only affects the nature of $3 D$ reconstruction, from projective to Euclidean reconstruction.

In the remaining part of this article, without explicit mention, the pair of projection matrices $\mathbf{P}$ and $\mathbf{P}^{\prime}$ considered is defined up to a projective transformation. This corresponds to the case described in Subsection II-B.3. We are therefore working in projective spaces which allow us to use some nice projective properties of quadric surfaces for conic reconstruction and correspondence. The Euclidean or others cases can be treated in exactly the same way by using the suitable projection matrices.

\section{Problem Formulation}

Given a corresponding pair of conics from two views

$$
\mathcal{C} \equiv \mathbf{u}^{T} \mathbf{C u}=0 \leftrightarrow \mathcal{C}^{\prime} \equiv \mathbf{u}^{T} \mathbf{C}^{\prime} \mathbf{u}^{\prime}=0,
$$

we require to find a conic in space which has been projected respectively into $\mathcal{C}$ and $\mathcal{C}^{\prime}$. A conic in space is generally represented as the complete intersection of a quadric surface and a plane. The reconstruction is therefore equivalent to finding the plane in which the conic lies, as we can take any one of the two cones associated with two conics in images as the quadric surface.

The cone equation associated with a given conic and a given view is obtained as follows.

Proposition 1: Given the projection matrix $\mathbf{P}$ of a camera, the equation of the cone which joins the conic $\mathbf{u}^{T} \mathbf{C u}=0$ in the image plane to the projection center of the camera is

$$
\mathbf{x}^{T} \mathbf{Q x}=0, \text { with } \mathbf{Q}=\mathbf{P}^{T} \mathbf{C P} .
$$

Proof: This is obtained by substituting $\lambda \mathbf{u}^{T}=\mathbf{P x}$ into the conic equation $\mathbf{u}^{T} \mathbf{C u}=0$. $\mathbf{x}^{T} \mathbf{Q x}=0$ is effectively a proper cone, for ${ }^{1}$

\footnotetext{
${ }^{1}$ For a quadric surface $\mathbf{x}^{T} \mathbf{A x}=0$, where $\mathbf{A}$ is $4 \times 4$ symmetric, the projective classification of quadric surfaces (cf. [20]) based on the rank of $\mathbf{A}$ is given as: a proper quadric surface has full rank 4 , a proper quadric cone rank 3 , a pair of distinct planes rank 2 and a repeated plane rank 1 .
} 
$\operatorname{rank}(\mathbf{Q})=\operatorname{rank}(\mathbf{P})=\operatorname{rank}(\mathbf{C})=3$ and $\operatorname{Ker}(\mathbf{Q})=$ $\operatorname{Ker}(\mathbf{P})$ which means that the vertex of the cone is the projection center of the camera.

The cones corresponding to the pair of conics are therefore

$$
\mathcal{Q} \equiv \mathrm{x}^{T} \mathbf{A x}=\mathbf{x}^{T} \mathbf{P}^{T} \mathbf{C P} \mathbf{x}=0
$$

and

$$
\mathcal{Q}^{\prime} \equiv \mathbf{x}^{T} \mathbf{B} \mathbf{x}=\mathbf{x}^{T} \mathbf{P}^{\prime T} \mathbf{C}^{\prime} \mathbf{P}^{\prime} \mathbf{x}=0
$$

in $\mathcal{P}^{3}$.

Consider the pencil of quadric surfaces $\mathcal{Q}+$ $\lambda \mathcal{Q}^{\prime}=0$, for every value of $\lambda$ the equation $\mathcal{Q}+\lambda \mathcal{Q}^{\prime}=0$ represents a quadric surface which passes through all the common points of $\mathcal{Q}$ and $\mathcal{Q}^{\prime}$. The points common to all quadric surfaces of the pencil are simply the points which make up the curve of intersection of $\mathcal{Q}$ and $\mathcal{Q}^{\prime}$, and this curve is the base curve of the pencil. The base curve of two quadric surfaces is generally a quartic curve. In our context, the reconstruction constraints impose that the corresponding cones intersect in a conic in space. As this conic in space should be part of the base curve, thus the base curve of the pencil should break up and one of the components is a conic in space! Even more, if one of the components of the base curve is a conic, the residual component should also be a conic. As a pair of planes can be considered as a degenerate quadric surface of rank 2 , according to the results of projective geometry (cf. [20]) on pencils of quadric surfaces, the degenerate quadric surface composed of the pair of planes belongs to the pencil of quadric surfaces in consideration. We are therefore led to examine a special pencil of quadric surfaces which contains a degenerated member of rank 2. Based on this observation, we can reformulate the problem of conic reconstruction as follows:

Proposition 2: The reconstruction of a conic in space from two views is equivalent to find a $\lambda$ such that the $\lambda$-matrix $\mathbf{C}(\lambda)=\mathbf{A}+\lambda \mathbf{B}$ has rank 2. The $\mathbf{x}^{T} \mathbf{A} \mathbf{x}=0$ and $\mathbf{x}^{T} \mathbf{B} \mathbf{x}=0$ are the proper cones corresponding to the two images of the conic in space.

We can also imagine that we may have the case where the pair of planes coincident, becoming a repeated plane which is a degenerate quadric surface of rank 1. If this were possible, the reconstruction would be essentially unique. However, it will be shown in the following proposition that this is impossible.

Proposition 3: If the conics in images are proper, there is no $\lambda$ such that $\mathbf{C}(\lambda)=\mathbf{A}+\lambda \mathbf{B}$ can have rank 1.

This proposition will be proved in the next section after other results have been introduced.

\section{POLYNOMIAL CONDITIONS FOR CORRESPONDENCE}

Unlike points and lines, two images of a conic in space contain sufficient information to impose correspondence conditions. The number of the independent conditions which is given by

Proposition 4: There exist only two independent polynomial conditions for a corresponding pair of conics.

Proof: From Proposition 2, we need only count the degrees of freedom of the rank 2 matrix and those of the matrix pencil. A $4 \times 4$ symmetric matrix up to a scaling factor counts for $10-1=9$ degrees of freedom, thus a general pencil counts for $9-1=8$ degrees of freedom. A rank 2 symmetric matrix $C$ of order 4 counts for 6 degrees of freedom, so there remain $2=8-6$ independent conditions.

We will now derive these two polynomial conditions.

Consider the characteristic polynomial of $\lambda$ matrix

$$
\mathbf{C}(\lambda)=\mathbf{A}+\lambda \mathbf{B},
$$

$|\mathbf{C}(\lambda)-\mu \mathbf{I}|=\mu^{4}+a_{1}(\lambda) \mu^{3}+a_{2}(\lambda) \mu^{2}+a_{3}(\lambda) \mu+a_{4}(\lambda)=0$.

As $\mathbf{C}(\lambda)$ is a real $4 \times 4$ symmetric matrix, for it to have rank 2 it must have two distinct nonzero eigenvalues and a double zero eigenvalue. The conditions we are looking for are equivalent to ${ }^{2}$

$$
\left\{\begin{array}{l}
a_{3}(\lambda)=0, \\
a_{4}(\lambda)=0
\end{array}\right.
$$

By definition, $a_{4}(\lambda)$ is the determinant of $\mathrm{C}(\lambda)$, therefore, it is the characteristic polynomial of the matrix pencil $\mathbf{A}+\lambda \mathbf{B}$, i.t.

$$
\begin{aligned}
& a_{4}(\lambda)=|\mathbf{C}(\lambda)|=|\mathbf{A}+\lambda \mathbf{B}|=I_{1} \lambda^{4}+I_{2} \lambda^{3}+I_{3} \lambda^{2}+I_{4} \lambda+I_{5}, \\
& { }^{2} \text { To be complete, we should also have } a_{2} \neq 0 \text { and } a_{1}^{2}-4 a_{2} \neq 0 \\
& \text { to guarantee two distinct nonzero eigenvalues. }
\end{aligned}
$$


where the coefficients $I_{j}$ are polynomials in the entries of $\mathbf{A}$ and $\mathbf{B}$.

Since both $\mathbf{A}$ and $\mathbf{B}$ have rank 3 , then $I_{1}=$ $|\mathbf{A}|=0$ and $I_{5}=|\mathbf{B}|=0$. The characteristic polynomial of the pencil is factorized as

$$
a_{4}(\lambda)=\lambda\left(I_{2} \lambda^{2}+I_{3} \lambda+I_{4}\right)=0
$$

There are generally four singular matrices of the pencil, each corresponds to one of the four generalized eigenvalues of the pencil, the roots of $a_{4}(\lambda)=0$. Two generalized eigenvalues of the pencil are easily read out as $\lambda=0$ and $\lambda=\infty$ which corresponds respectively to $\mathbf{A}$ and $\mathbf{B}$. The two others are the solutions of the quadratic equation

$$
I_{2} \lambda^{2}+I_{3} \lambda+I_{4}=0 .
$$

Before going ahead, let us recall the following known result [21]:

Lemma 1: Given symmetric matrices $\mathbf{A}$ and $\mathbf{B}$ of order $n$. If $\lambda_{i}$ is a generalized eigenvalue of $\mathbf{A}+\lambda \mathbf{B}$ of multiplicity $k_{i}$, then $k_{i}$ is at least $n-m$ for $\mathbf{C}\left(\lambda_{i}\right)=\mathbf{A}+\lambda_{i} \mathbf{B}$ to have rank $m$.

Proof: This is a direct consequence of the fact that the dimension of nullspace of $\mathbf{A}+\lambda_{i} \mathbf{B}$ can not exceed $k_{i}$.

In order to have a rank 2 matrix in the pencil, we should at least have a generalized eigenvalue of multiplicity 2 , hence the above quadratic equation (3) must have two equal roots. The condition for this is

$$
\Delta \equiv I_{3}^{2}-4 I_{2} I_{4}=0,
$$

which is actually the first condition for correspondence.

It is now straightforward that we cannot have a rank 1 matrix in this matrix pencil. Thus we must at least have a triple generalized eigenvalue which is obviously impossible for we have already two distinct ones 0 and $\infty$. This proves Proposition 3 .

After $a_{4}(\lambda)=0$ of $(2)$ is examined, we can now examine $a_{3}(\lambda)=0$. The $a_{3}(\lambda)$ is a cubic polynomial in $\lambda$, it can be written as

$$
a_{3}(\lambda)=J_{1} \lambda^{3}+J_{2} \lambda^{2}+J_{3} \lambda+J_{4},
$$

where $J_{i}$ are polynomials in the entries of $\mathbf{A}$ and B.
The second condition is derived by computing the resultant of $a_{3}(\lambda)$ and $a_{4}(\lambda)$ with respect to $\lambda$. The explicit form of this resultant, further simplified by using $\Delta=0$, gives the second polynomial condition

$$
\Theta \equiv-J_{1} I_{3}^{3}+2 J_{2} I_{3}^{2} I_{2}-4 J_{3} I_{3} I_{2}^{2}+8 J_{4} I_{2}^{3}=0 .
$$

This completes the derivation of two correspondence conditions which are respectively $\Delta=0$ and $\Theta=0$.

\section{A. Remarks on the correspondence conditions}

A.1 Algebraic implication of $\Delta=0$ and $\Theta=0$

From an algebraic point of view (cf. [22], [21], $[23])$, we are dealing with a special kind of matrix pencils $\mathbf{C}(\lambda)=\mathbf{A}+\lambda \mathbf{B}$ which have a double generalized eigenvalue. It is known that the existence of multiple eigenvalues (generalized or not) is generally a complicating factor. Matrix pencils in which multiple generalized eigenvalues occur may be divided into two classes according to whether or not the dimensions of the null space (i.e. its geometric multiplicity ${ }^{3}$ ) equal its algebraic multiplicity. When the algebraic multiplicity exceeds the geometric multiplicity, the matrix pencil is defective ${ }^{4}$. Otherwise, the matrix pencils are simple, non-defective.

For simple matrix pencils, $k_{i}=n-m$ instead of $k_{i} \geq n-m$ in Lemma 1 , the condition $\Delta=0$ is therefore becoming necessary and sufficient for $C$ to have rank 2 in our problem. The second condition $\Theta=0$ is becoming obselete. It is in fact equivalent to the condition requiring matrix pencils to have simple structures. Fortunately, the matrix pencils from the physically significant problems are almost exclusively of the class having the simple structure [21]. Therefore from the practical point of view, we can reasonably assume that the matrix pencil from two views of the same conic has a simple structure. In this case, the first condition $\Delta=0$ is a necessary and sufficient condition for conic correspondence. The second condition is generally implied by $\Delta=0$ under the assumption of simple structure. Another consequence of this consideration is that the use of the second condition even for other purposes, for instance, mo-

\footnotetext{
${ }^{3}$ The number of linearly independent eigenvectors associated with this multiple eigenvalue.

${ }^{4}$ Or as being derogatory, they are non-diagonalizable.
} 
tion estimation from conics should be moderated in practice as well. The algorithms which use this condition as constraints will no doubt cause numerical instability.

Interestingly, $\Delta=0$ admits also a nice invariant interpretation that is given in the following subsection.

\section{A.2 Invariant interpretation of $\Delta$}

Proposition 5: The condition $\Delta=0$ can be interpreted as that the absolute projective invariant $I$ associated with the pair of cones is a constant.

Proof: Let us first consider the invariant algebra $^{5}[20]$, [25], [9] of the pair of the quadratic forms $\mathbf{x}^{T} \mathbf{A} \mathbf{x}$ and $\mathbf{x}^{T} \mathbf{B} \mathbf{x}$, the determinant of the pair of the quadratic forms:

$|\lambda \mathbf{A}+\mu \mathbf{B}|=I_{1} \lambda^{4}+I_{2} \lambda^{3} \mu+I_{3} \lambda^{2} \mu^{2}+I_{4} \lambda \mu^{3}+I_{5} \mu^{4}$.

When $\mathrm{x}$ transforms into $\mathrm{x}^{\prime}=\mathbf{T} \mathrm{x}$ by any non singular space collineation $\mathbf{T}, I_{j}$ are transformed into $I_{j}^{\prime}=|\mathbf{T}|^{2} I_{j} . I_{j}, j=1, \ldots, 5$ are relative invariants of weight 2 of the two quaternary quadratic forms $\mathbf{x}^{T} \mathbf{A} \mathbf{x}$ and $\mathbf{x}^{T} \mathbf{B} \mathbf{x}$.

Now consider the invariant algebra of a pair of quadric surfaces $\mathbf{x}^{T} \mathbf{A} \mathbf{x}=0$ and $\mathbf{x}^{T} \mathbf{B} \mathbf{x}=0$ which is associated with a family of quadratic forms $\mathbf{x}^{T}(\lambda \mathbf{A}) \mathbf{x}$ and $\mathbf{x}^{T}(\mu \mathbf{B}) \mathbf{x}$. The relative invariants are related as follows:

$$
\begin{aligned}
& I_{1}(\lambda, \mu)=\lambda^{4} I_{1}, \\
& I_{2}(\lambda, \mu)=\lambda^{3} \mu I_{2}, \\
& I_{3}(\lambda, \mu)=\lambda^{2} \mu^{2} I_{3}, \\
& I_{4}(\lambda, \mu)=\lambda \mu^{3} I_{4}, \\
& I_{5}(\lambda, \mu)=\mu^{4} I_{5} .
\end{aligned}
$$

As we are considering a pair of cones, so $I_{1}=$ $I_{5}=|\mathbf{A}|=|\mathbf{B}|=0$. We are left with nonzero $I_{2}$, $I_{3}$ and $I_{4}$. While taking into account the power degrees of $\lambda$ and $\mu$, the unique absolute invariant of a pair of the cones is

$$
I=\frac{I_{3}^{2}}{I_{2} I_{4}}
$$

As $\Delta=I_{3}^{2}-4 I_{2} I_{4}$, therefore

$$
\Delta=0 \Leftrightarrow I=4 \text {. }
$$

\footnotetext{
${ }^{5}$ For a more general introduction to invariants, on can refer to $[5],[24]$.
}

\section{Closed FORM SOLUTION OF RECONSTRUCTION}

In this section, we will be developing a closed form solution for reconstructing the conic in space from its two views identified as a corresponding pair. According to our reformulation of the problem, we first have to determine the degenerate quadric surface, then extract the planes from it.

\section{A. Computation of the degenerate quadric surface}

Since we must have two equal roots for the quadratic equation (3), the double generalized eigenvalue is directly obtained by

$$
\lambda=-\frac{I_{3}}{2 I_{2}} .
$$

Then we obtain the matrix $\mathbf{C}=\mathbf{A}+\lambda \mathbf{B}$ of the degenerate quadric surface. The remaining effort for conic reconstruction requires only the extraction of the two planes from $\mathbf{C}$, a rank 2 matrix.

\section{B. Extraction of the plane pair}

The extraction of the plane pair from $\mathbf{C}$ consists of the eigen analysis of $\mathrm{C}$, which can be directly solved as follows.

Going back to the characteristic polynomial of the matrix $\mathbf{C}(\lambda)$, it is simplified by the second condition $\Theta=0$ as

$$
\mu^{2}\left(\mu^{2}+a_{1}(\lambda) \mu+a_{2}(\lambda)\right)=0 .
$$

The remaining two nonzero eigenvalues $\mu_{1}$ and $\mu_{2}$ are the roots of the quadratic equation:

$$
\mu^{2}+a_{1}(\lambda) \mu+a_{2}(\lambda)=0 .
$$

As $\mathbf{C}$ is a real symmetric matrix, there exists a non singular transformation $\mathbf{T}$ such that $\mathbf{C}$ is diagonalized:

$$
\mathbf{T}^{T} \mathbf{C} \mathbf{T}=\operatorname{diag}\left(\mu_{1}, \mu_{2}, 0,0\right) .
$$

The quadric surface $\mathbf{x}^{T} \mathbf{C x}=0$ is therefore transformed by $\mathbf{x}=\mathbf{T x}^{\prime}$ into $\mathbf{x}^{T} \operatorname{diag}\left(\mu_{1}, \mu_{2}, 0,0\right) \mathbf{x}^{\prime}=0$, i.e.

$$
\mu_{1} x^{\prime 2}+\mu_{2} y^{\prime 2}=0 .
$$

The pair of planes $\pi_{i}^{\prime} \equiv \mathbf{p}_{i}^{\prime T} \mathbf{x}^{\prime}=0, i=1,2$ in the transformed reference frame is 
$\left(\sqrt{\mu_{1}}, \pm \sqrt{-\mu_{2}}, 0,0\right)^{T} \mathbf{x}^{\prime}=\sqrt{\mu_{1}} x^{\prime} \pm \sqrt{-\mu_{2}} y^{\prime}=0$.

It is obvious that to obtain real planes, we must have $^{6}$

$$
a_{2}(\lambda)=\mu_{1} \mu_{2}<0
$$

Let $\mathbf{v}_{1}$ and $\mathbf{v}_{2}$ be the eigenvectors corresponding to the eigenvalues $\mu_{1}$ and $\mu_{2}$ of $C$. The plane pair $\pi_{i} \equiv \mathbf{p}_{i}^{T} \mathbf{x}=0, i=1,2$ in the original reference frame are obtained by

$$
\left(\mathbf{T} \mathbf{p}_{i}^{\prime}\right)^{T} \mathbf{x}=\left(\sqrt{\mu_{1}} \mathbf{v}_{1} \pm \sqrt{-\mu_{2}} \mathbf{v}_{2}\right)^{T} \mathbf{x}=0 .
$$

Then the conic in space is defined as the intersection of one of the two cones with the plane recovered above:

$$
\left\{\begin{array}{l}
\mathbf{x}^{T} \mathbf{A x}=0 \\
\mathbf{p}_{i}^{T} \mathbf{x}=0
\end{array}\right.
$$

C. Determination of the unique plane from the plane pair

At this stage, the reconstruction of conics in space is ambiguous up to two solutions, defined by any one of the plane pair. We now show that this ambiguity may be removed in some special cases.

Let us first restrict ourself to the Euclidean case, i.e. the projection matrices are either from calibration or from motion cases. If we further assume that the conic in space is a non-transparent object (a wired conic in space is a typical example of transparency), the visibility constraint may be used to get rid of the surplus solution. To be visible for a non-transparent object from two different viewpoints, it is necessary that the two viewpoints be located on the same side of the plane. Look at Figure 1 in which one of planes of the pair is in between the two viewing centers. This plane apparently cannot be the solution of the problem if the conic in space is not transparent.

In practice, this can be easily checked as follows. The projection centers of the two views, given their projection matrices $\mathbf{P}=\left(P_{3 \times 3} p\right)$ and $\mathbf{P}^{\prime}=\left(P_{3 \times 3}^{\prime} p^{\prime}\right)$ are

$$
\mathbf{o}=\operatorname{Ker}(\mathbf{P}) \quad \text { and } \quad \mathbf{o}^{\prime}=\operatorname{Ker}\left(\mathbf{P}^{\prime}\right) .
$$

\footnotetext{
${ }^{6}$ This inequality condition does not affect the degree of freedom of the matrix pencil, so it does not affect the Proposition 4.
}

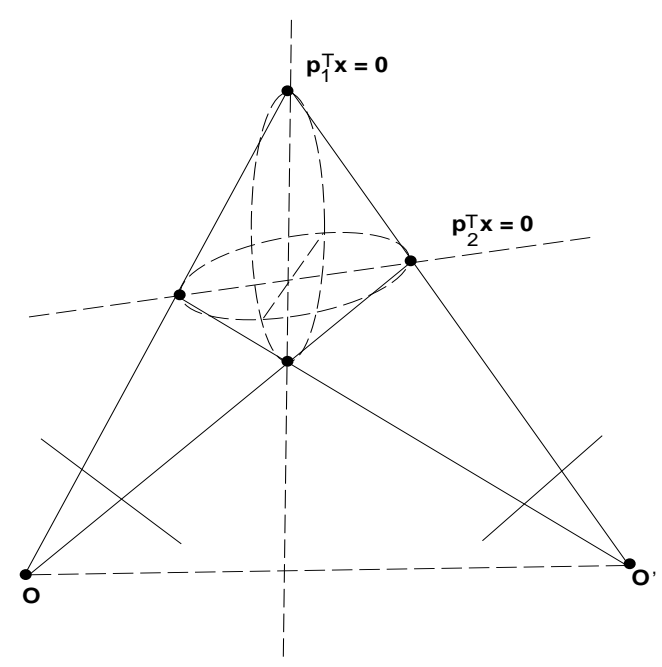

Fig. 1. This figure illustrates that one of the planes of the pair is always between two camera centers, therefore it is not the solution of the problem if we are considering non-transparent objects. One side of the plane $p_{2}^{T} x=0$ is visible for both cameras, however each side of the plane $p_{1}^{T} x=0$ is visible by a different camera.

If $\left(\mathbf{o}^{T} \mathbf{p}_{i}\right)\left(\mathbf{o}^{T T} \mathbf{p}_{i}\right)>0$ then $\mathbf{o}$ and $\mathbf{o}^{\prime}$ lie both on the same side of the plane $\mathbf{p}_{i}^{T} \mathbf{x}=0$, otherwise they lie on different sides of the plane. This test allows us to remove the reconstruction ambiguity for non-transparent conics.

It can also be noted that this test can be extended to the projective case if we further assume that conics contain no real points at infinity (i.e. only ellipses) because the visibility is still valid for objects containing no points at infinity in the weak calibration case [26], [27].

\section{Summary of the computation}

After the conic features have been extracted from each of two views, the correspondences of conics through two views are established by the following procedure.

1. For each pair of conics $\mathcal{C}_{i}$ and $\mathcal{C}_{j}^{\prime}$,

- form the cones $\mathbf{A}=\mathbf{P}^{T} \mathbf{C}_{i} \mathbf{P}$ and $\mathbf{B}=$ $\mathbf{P}^{\prime T} \mathbf{C}_{j}^{\prime} \mathbf{P}^{\prime}$

- compute $I_{2}, I_{3}$ and $I_{4}$, then $\Delta_{i, j}$.

2. A potential corresponding pair of conics is selected as the pair that has the smallest absolute value of $\Delta_{i, j}$ among all possible pairs of conics between two views.

3. If $\left|\Delta_{i, j}\right|<\epsilon$, it is taken to be a corresponding pair, otherwise there is no correspondence for the given $C_{i}$. 
For each corresponding pair of conics, the conic in space is reconstructed by the following procedure.

1. Compute $\lambda=-I_{3} / 2 I_{2}$;

2. Compute the degenerate quadric from the $\lambda$, $\mathbf{C}=\mathbf{A}+\lambda \mathbf{B}$ :

3. Extract the two planes

(a) If $a_{2}>0$, solve the quadratic equation (4) for $\mu_{1}$ and $\mu_{2}$, otherwise no real solution possible.

(b) Compute the eigenvectors $\mathbf{v}_{1}$ and $\mathbf{v}_{2}$ associated with $\mu_{1}$ and $\mu_{2}$ by solving the linear equation

$$
\left(\mathbf{C}-\mu_{i} \mathbf{I}\right) \mathbf{v}_{i}=0 .
$$

(c) The plane pair is given as

$$
\left(\sqrt{\mu_{1}} \mathbf{v}_{1} \pm \sqrt{-\mu_{2}} \mathbf{v}_{2}\right)^{T} \mathbf{x}=0
$$

4. For non-transparent objects, select the plane for which $\left(\mathbf{o}^{T} \mathbf{p}_{i}\right)\left(\mathbf{o}^{T} \mathbf{p}_{i}\right)$ is positive.

\section{EXPERIMENTAL RESULTS}

The theoretical results presented above for corresponding conditions and reconstruction of conics have been implemented. The accuracy, stability of reconstruction and the discriminality of correspondence conditions are studied both for simulated and real images.

\section{A. The experiments with simulated images}

\section{A.1 Simulation set-up}

- We use the calibration matrices from a real stereo system. They are given by

$$
\begin{aligned}
\mathbf{P} & =\left(\begin{array}{cccc}
1.393757 & -0.244708 & -14.170794 & 368.0 \\
10.624195 & 2.396275 & -0.433595 & 202.0 \\
0.002859 & 0.011811 & -0.003481 & 1.0
\end{array}\right) \\
\mathbf{P}^{\prime} & =\left(\begin{array}{cccc}
1.374060 & -0.612998 & -14.189693 & 371.0 \\
10.979978 & -1.621189 & -0.469463 & 207.0 \\
0.007648 & 0.010572 & -0.003449 & 1.0
\end{array}\right)
\end{aligned}
$$

- We view with these two cameras two conics in space described respectively by the intersection of the quadric surface $\mathbf{x}^{T} \mathbf{Q}_{1} \mathbf{x}=0$ and the plane $\mathbf{p}_{1}^{T} \mathbf{x}=0$ for the first conic in space and the intersection of $\mathbf{x}^{T} \mathbf{Q}_{2} \mathbf{x}=0$ and $\mathbf{p}_{2}^{T} \mathbf{x}=0$ for the second. The plane $\mathbf{p}_{2}^{T} \mathbf{x}=0$ is taken as being parallel to the first image plane and goes through the center of $\mathbf{x}^{T} \mathbf{Q}_{2} \mathbf{x}=0$.

$$
\left\{\begin{array}{c}
\mathbf{Q}_{1}=\left(\begin{array}{cccc}
-0.0013 & 0.4710^{-5} & -0.00023 & 0.0058 \\
0.4710^{-5} & -0.000078 & -0.00034 & 0.0033 \\
-0.00023 & -0.00034 & -0.0014 & 0.011 \\
0.0058 & 0.0033 & 0.011 & -0.038
\end{array}\right) \\
\mathbf{p}_{1}=(-0.021,-0.16,-0.092,1.0)^{T}
\end{array}\right.
$$

$$
\left\{\begin{aligned}
\mathbf{Q}_{2} & =\left(\begin{array}{cccc}
1.0 & 0.0 & 0.0 & -9.0 \\
0.0 & 1.0 & 0.0 & -2.0 \\
0.0 & 0.0 & 1.0 & -10.0 \\
-9.0 & -2.0 & -10.0 & 85.0
\end{array}\right) \\
\mathbf{p}_{2} & =(-0.196589,-0.812143,0.239359,1.0)^{T}
\end{aligned}\right.
$$

- The conic in space is analytically projected into image planes by the two calibration matrices.

- The projected conics in images are resampled as a list of points. Each location of resampled points is perturbed by varying levels of pixel noise of a uniform distribution.

- Each list of perturbed points is then fitted to a conic of form

$$
a x^{2}+b x y+c y^{2}+d x+e y+f=0 .
$$

With the normalization $f=1$, least squares fitting can be easily implemented as a linear minimization procedure. Note that using this normalization directly fails for all conics through the origin (as in this case $f=0$ ), but this can be easily overcome by shifting the data away from the origin if necessary. In practice, very good results are obtained using this normalization. More sophisticated fitting algorithms are only necessary when a small part of curve data is visible and/or the curve data is very scattered. A more detailed description on conic fitting can be found in [2], [28].

A.2 Reconstruction stability w.r.t. pixel errors

Conic reconstruction with respect to different pixel errors of image points is performed to demonstrate its stability. Table I and II show the numerical results for the two conics in space. As conic reconstruction is ambiguous, the solution for the plane which is closer to the known space plane of the conic is marked in bold font. Numerically, we can never have a perfect rank 2 matrix $\mathbf{C}$. It is well known that numerical rank is nicely characterized in terms of SVD [22]. In the tables of results, we 
also computed the ratio $\sigma_{3} / \sigma_{2}$ of the second largest $\sigma_{2}$ and the third largest singular values $\sigma_{3}$, which indicates how near $C$ is to a rank 2 matrix. The closer to zero $\sigma_{3} / \sigma_{2}$ is, the nearer $C$ is to rank 2 .

We notice that for both space conics, the degradation with the increasing pixel noise is extremely graceful. This is largely due to the fact that conics are global primitives and small pixel errors are very well corrected by the fitting process.

\section{A.3 Discriminality of correspondence condition}

To demonstrate the discriminality of correspondence conditions, a set of very similar conics is generated by deforming the initial one. The deformation is performed by adding different levels of pixel perturbation to the discreted conic points, then fitting the disturbed points to get the new conic. The closeness of the set of the conics generated in the second image can be deduced from Figure 2 in which four of them are displayed.

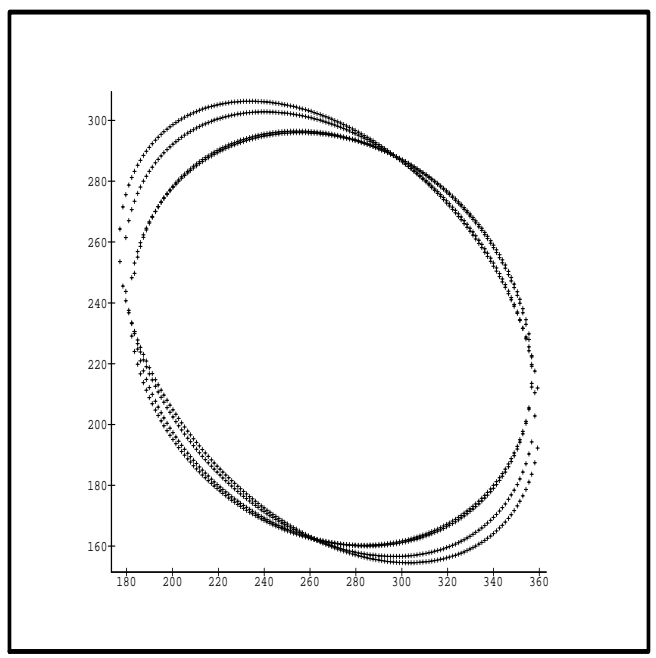

Fig. 2. The four conics of the set of generated similar conics. If we look at conics from right to left in the down-left part of the conics, they correspond to $C_{4}$, $C_{0}, C_{5}$ and $C_{6}$.

Table III shows the computed $\Delta$ for each pair of conics. The absolute value of $\Delta$ increases with the increasing discrepancy of conic pairs. Note that as $C_{1}$ is a slightly deformed version of $C_{0}$, and $C_{1}^{\prime}$ of $C_{0}^{\prime}$, it is quite reasonable that $C_{1}$ is as close to $C_{0}^{\prime}$ as to $C_{1}^{\prime}$, as suggested in the table.

\section{B. The experiments with real images}

B.1 Wooden house images

We first used a wooden house image sequence that has been frequently used for self-calibration. Each camera position is calibrated with respect to a coordinate frame associated to the objects in view. Figure 3 illustrates one of the images in which we can see the calibration coordinate frame associated with the scene and the three conics used for experimentation. The stereo pair of images appear in Figure 4 in which their contour images are displayed.

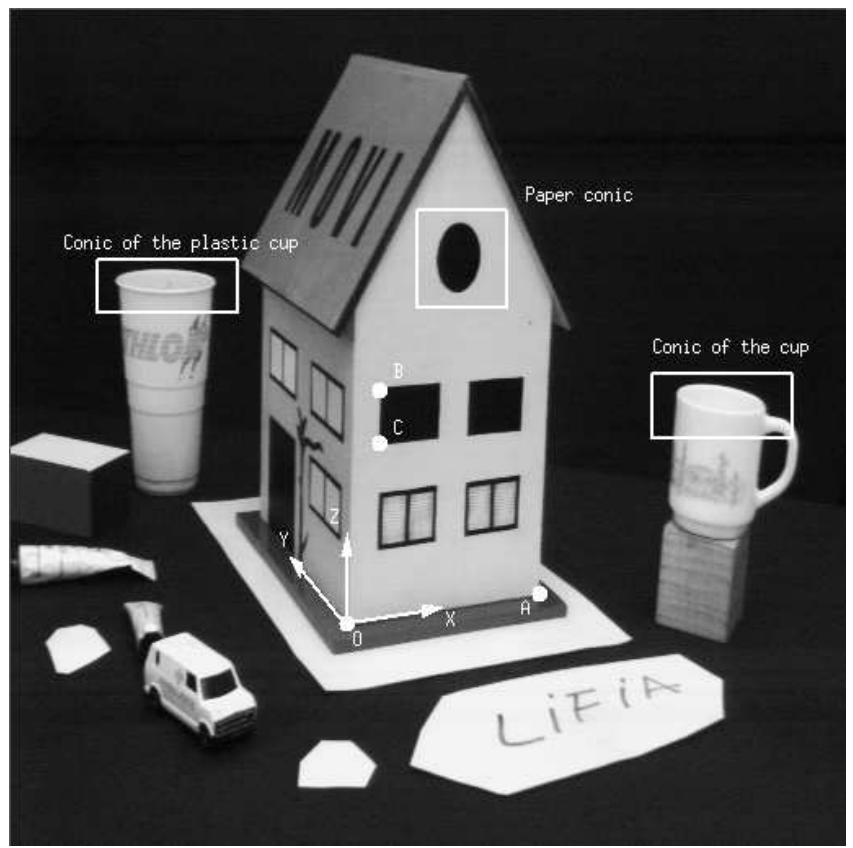

Fig. 3. One of the two original images in which the coordinate system for calibration and the conics used in the experimentation are marked. To have an idea of the real size of the object, $O A$ and $B C$ are measured as $13.75 \mathrm{~cm}$ and $2.5 \mathrm{~cm}$.
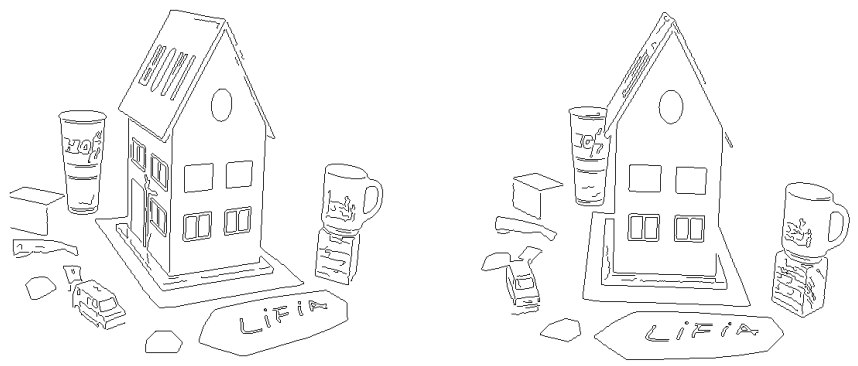

Fig. 4. The contour images of the stereo pair of images.

The pair of images is treated by a Canny-like edge detector, and then linked into contour chains. 
TABLE I

RECONSTRUCTION RESUlTS FOR THE FIRST SIMULATED CONIC IN SPACE WITH DIFFERENT PIXEL ERRORS FOR IMAGE POINTS BEFORE FItTING. $\sigma_{2}$ AND $\sigma_{3}$ ARE THE SECOND AND THE thiRd LARgEST Singular VALUes of the Plane

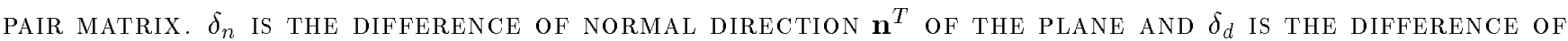
Distance $d$ OF THE Plane With THaT of THE KNOWN PLANE.

\begin{tabular}{|c|c|c|c|c|c|c|}
\hline Noise & $\Delta$ & $\Theta$ & $\sigma_{3} / \sigma_{2}$ & Plane pair $\left(\mathbf{n}^{T},-d\right)$ & $\delta_{n}$ & $\delta_{d}$ \\
\hline \pm 1.5 & $0.710^{-5}$ & $0.710^{-10}$ & $0.3910^{-2}$ & $\begin{array}{c}\mathbf{- 0 . 1 1 5 4 8 7 ,}-\mathbf{- 0 . 8 6 1 9 9 1 ,}, \mathbf{- 0 . 4 9 3 5 9 3 , ~ 5 . 3 8 1 5 0 )} \\
(-0.970367,0.240090,0.0273005,2.25818)\end{array}$ & 0.17 & 0.002 \\
\hline \pm 2.5 & $-0.310^{-4}$ & $0.1310^{-10}$ & $0.2610^{-2}$ & 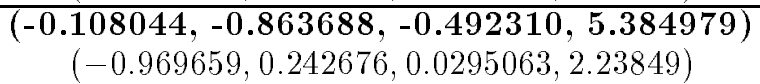 & 0.36 & 0.002 \\
\hline \pm 3.5 & -0.5 & 0 & $0.7210^{-2}$ & 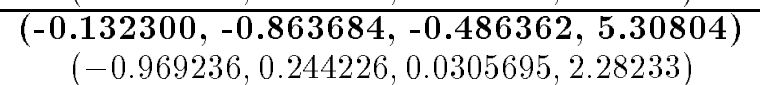 & 1.2 & 0.08 \\
\hline \pm 4.5 & 0 & 1 & 0.024 & 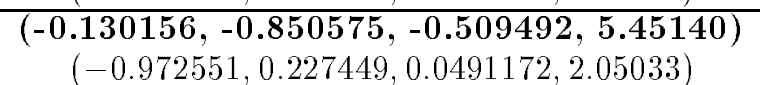 & 1.42 & 0.07 \\
\hline
\end{tabular}

TABLE II

RECONSTRUCTION RESUlTS FOR THE SECOND SIMUlATED CONIC IN SPACE WITH DIFFERENT PIXEL ERRORS FOR IMAGE POINTS BEFORE FITTING.

\begin{tabular}{|c|c|c|c|c|c|c|}
\hline Noise & $\Delta$ & $\Theta$ & $\sigma_{3} / \sigma_{2}$ & Plane pair $\left(n^{T},-d\right)$ & $\delta_{n}$ & $\overline{\delta_{d}}$ \\
\hline \pm 1.5 & $-0.910^{-7}$ & $-0.2010^{-12}$ & $0.1810^{-3}$ & $\begin{array}{c}\mathbf{( - 0 . 2 2 7 5 2 7 ,} \mathbf{- 0 . 9 3 4 7 6 1 , 0 . 2 7 2 8 6 1 , ~ 1 . 1 7 5 7 1 )} \\
(-0.954314,0.298090,-0.0206877,8.02997)\end{array}$ & 0.17 & 0.03 \\
\hline \pm 2.5 & $-0.910^{-7}$ & $0.1610^{-11}$ & $0.3010^{-3}$ & 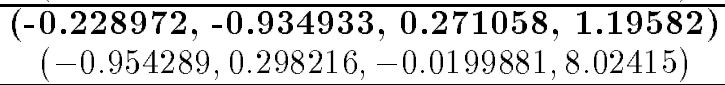 & 0.30 & 0.05 \\
\hline \pm 3.5 & $0.1610^{-6}$ & $0.5010^{-11}$ & $0.5110^{-3}$ & $\begin{array}{c}\mathbf{( - 0 . 2 3 0 8 4 8 ,}-\mathbf{- 0 . 9 3 5 0 1 7}, \mathbf{0 . 2 6 9 1 6 9}, \mathbf{1 . 2 1 8 4 0}) \\
(-0.954243,0.298441,-0.0188021,8.01351)\end{array}$ & 0.45 & 0.07 \\
\hline \pm 4.5 & $0.910^{-6}$ & $0.1010^{-10}$ & $0.9210^{-3}$ & $\begin{array}{c}\mathbf{( - 0 . 2 3 3 1 5 4 ,}-\mathbf{- 0 . 9 3 5 0 0 7 , ~ 0 . 2 6 7 2 1 0 , ~ 1 . 2 4 3 2 8 )} \\
(-0.954174,0.298761,-0.0171345,7.99810)\end{array}$ & 0.62 & 0.09 \\
\hline
\end{tabular}

TABLE III

The computed $\Delta$ for each pair of CONics $C_{i}$ And $C_{j}^{\prime}, C_{i}$ is a CONic of the first image and $C_{j}^{\prime}$ a CONIC OF THE SECOND IMAGE.

\begin{tabular}{|c|c|c|c|c|c|c|c|}
\hline$\Delta$ & $C_{0}^{\prime}$ & $C_{1}^{\prime}$ & $C_{2}^{\prime}$ & $C_{3}^{\prime}$ & $C_{4}^{\prime}$ & $C_{5}^{\prime}$ & $C_{6}^{\prime}$ \\
\hline$C_{0}$ & $\mathbf{0 . 2 0 1 0 ^ { - 1 5 }}$ & $-0.6210^{-5}$ & $-0.8210^{-4}$ & $-0.4710^{-4}$ & $0.2410^{-3}$ & $0.5010^{-2}$ & $0.2610^{-1}$ \\
\hline$C_{1}$ & $\mathbf{- 0 . 4 6 1 0 ^ { - 5 }}$ & $\mathbf{0 . 6 6 1 0 ^ { - 5 }}$ & $-0.4210^{-4}$ & $-0.8110^{-4}$ & $0.1810^{-3}$ & $0.5110^{-2}$ & $0.2610^{-1}$ \\
\hline
\end{tabular}

The contour chains of the three conics: the paper conic, the conic of the cup and the conic of the plastic cup, are selected by hand from the contour chains. For automatic selection of contour chains of conics, one may refer to [29]. The selected contour chains are then fitted by the procedure described above.

The correspondences of conics through two images are unambiguously established by comparing the computed $\Delta$ of Table IV.

The reconstruction results are illustrated in Table V. To gain an idea of the reconstruction results, each plane equation is put in the form $\left(\mathbf{n}^{T},-d\right)$ where $\mathbf{n}^{T}$ is the unit normal direction vector of the plane and $d$ is the distance of the origin to the plane. The plane which corresponds to the real solution, checked by applying the visibility test, is marked in bold font in the plane pair. Recall that the coordinate frame for calibration is illustrated in Figure 3. The paper conic lies in the vertical plane $y=0$, the conics of the cup and the plastic cup lie almost in the planes parallel to the horizontal plane, and the plastic cup is little higher than the cup. All these facts are confirmed by the reconstruction results. 
TABLE $\mathrm{V}$

THE ReCONSTRUCtion RESUlts of THE THREE CONICS OF THE WOODEN House images.

\begin{tabular}{|c|c|c|c|c|}
\hline & $\Delta$ & $\Theta$ & $\overline{\sigma_{3} / \sigma_{2}}$ & Plane pair $\left(n^{T},-d\right)$ \\
\hline paper & -0.02 & $-0.510^{-9}$ & 0.032 & $\begin{array}{c}(\mathbf{- 0 . 0 7 0 7 ,} \mathbf{0 . 9 9 3 0 , 0 . 0 9 4 4 5 , - 0 . 4 8 4 5 )} \\
(0.9501,0.3115,-0.01362,-5.855)\end{array}$ \\
\hline cup & -0.1 & $-0.110^{-9}$ & 0.018 & $\begin{array}{c}\mathbf{( - 0 . 0 5 6 4 5 ,}, \mathbf{- 0 . 0 3 2 8 6 , 0 . 9 9 7 9 ,}-\mathbf{- 9 . 4 8 6 )} \\
(0.6811,-0.2470,0.6893,-26.16)\end{array}$ \\
\hline plastic & -0.6 & $-0.1410^{-8}$ & 0.34 & 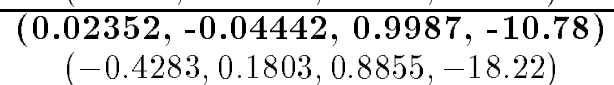 \\
\hline
\end{tabular}

TABLE IV

RESUlts OF THE COMPUTATION OF $\Delta$ IN ORDER TO ESTABLISH THE CORRESPONDENCES OF THE CONICS. THE RAW ENTRIES CORRESPOND TO THE CONICS OF THE FIRST IMAGE AND THE COLUMN ENTRIES TO THOSE OF THE SECOND IMAGE.

\begin{tabular}{|c|c|c|c|}
\hline$\Delta$ & paper & cup & plastic \\
\hline paper & $\mathbf{- 0 . 0 2}$ & 358. & -0.9 \\
\hline cup & 2595. & $\mathbf{- 0 . 1}$ & 1034. \\
\hline plastic & $\mathbf{- 2 8 .}$ & 5214. & $\mathbf{- 0 . 6}$ \\
\hline
\end{tabular}

\section{B.2 Breakfast images}

We then used a real stereo system coupled to a robot, the stereo system is calibrated off-line with a special calibration objet. The image pair of Figure 5 is taken by this stereo system. The process from edge detection to conic fitting is the same as in the above example. The fitted conics are shown in Figure 6.
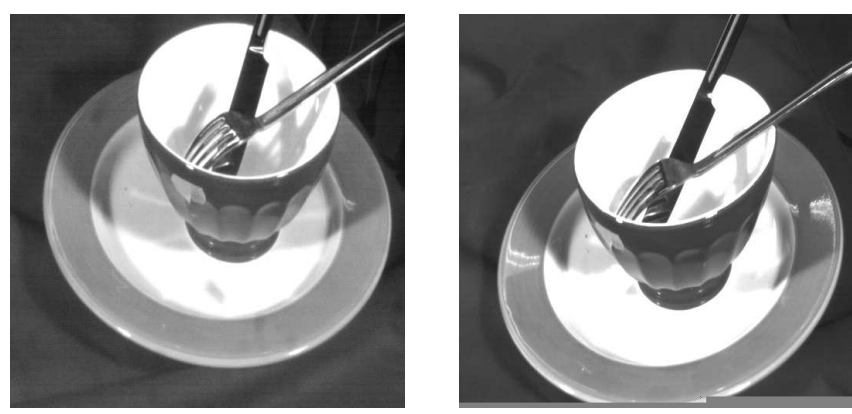

Fig. 5. The initial stereo pair of breakfast images.

The correspondences are unambiguously established based on the computed $\Delta \mathrm{s}$, shown in $\mathrm{Ta}^{-}$ ble VI.

Table VII shows the reconstruction results. To have a rough idea of the reconstruction quality, the heights of the conics from the ground, measured with a ruler, are respectively $8.5 \mathrm{~cm}$ for the bowl, $3.0 \mathrm{~cm}$ for the dish outside and $2.3 \mathrm{~cm}$ the dish in-

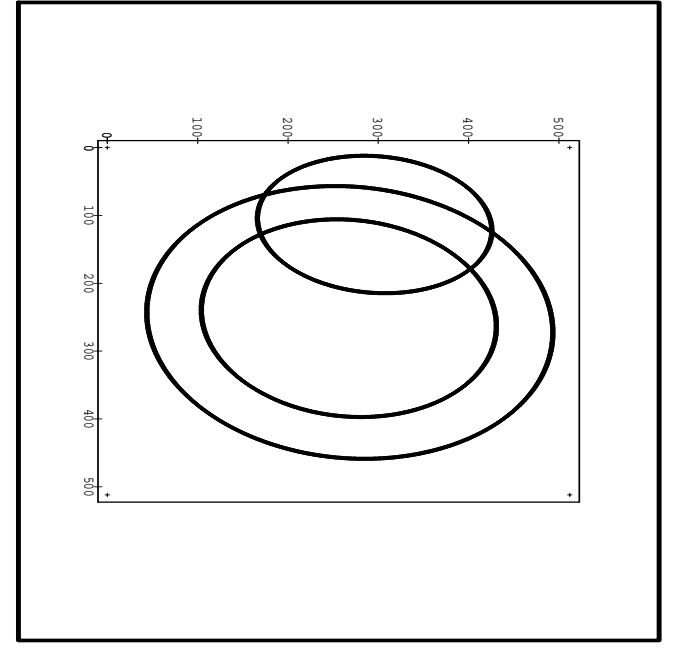

Fig. 6. Fitting of three conics in one of the breakfast images.

TABLE VI

THE COMPUTATION OF $\Delta$ FOR EACH PAIR OF CONICS OF two images. The RaW entries correspond to the CONICS OF THE FIRST IMAGE AND THE COLUMN ENTRIES TO THOSE OF THE SECOND IMAGE.

\begin{tabular}{|c|c|c|c|}
\hline & bowl & dish inside & dish outside \\
\hline bowl & $\mathbf{- 0 . 0 0 1}$ & -15.0 & -3.7 \\
\hline dish inside & -6.0 & $\mathbf{- 0 . 0 0 0 1}$ & 0.54 \\
\hline dish outside & -9.4 & 3.8 & $\mathbf{- 0 . 0 0 0 5}$ \\
\hline
\end{tabular}

side. That makes a difference of $5.5 \mathrm{~cm}$ between the bowl and the dish outside borders and $0.7 \mathrm{~cm}$ between the inside and outside of the dish. Obviously the planes on which conics lie should all be parallel to the ground. The computed difference in the heights are $5 \mathrm{~cm}$ for $5.5 \mathrm{~cm}$ and $0.8 \mathrm{~cm}$ for $0.7 \mathrm{~cm}$. The difference of plane orientations are $2.6^{\circ}$ between the bowl and the inside and $1.7^{\circ}$ between the inside and the outside border.

Quantitative conclusions can not be drawn from this due to inaccuracy of camera calibration and irregularity of objects. Another important factor 
is that the porcelain objects have smoothed borders and so their image contours have the effect of a moving tangential contour.

Notice that in this experiment the computed $\Theta$ in Table VII are much higher than in the previous examples. However the ratios of singular values are small enough to indicate the numerical rank of the matrix $C . \Theta$ is related to the scale of the problems.

\section{CONCLUSION}

We have proposed a solution to conic correspondence and conic reconstruction from two images within a unified framework for both the projective and the Euclidean case. We derived two polynomial conditions $\Delta=0$ and $\Theta=0$ to establish the correspondences of conics. We have shown algebraically that the first condition $\Delta=0$ is more important than the second one from the practical point of view. We also proposed a simple analytical method for reconstructing the corresponding conics in space. It is also shown that the conic reconstruction is generally ambiguous up to two solutions, and only unique for non-transparent objects.

The method shown is simpler and more stable than existing methods, as the intrinsic properties of the problem are fully exploited. The experimental results based on both simulated and real images confirm that $\Delta$ is a discriminative correspondence criterion and the reconstruction method is accurate and numerically stable.

\section{ACKNowledgements}

This work is partly supported by European Esprit BRA projects Viva which is gratefully acknowledged. We would also like to thank R. Mohr, R. Horaud, F. Veillon, Z. Lan and D. Morris for providing interesting discussion and help.

\section{REFERENCES}

[1] K. Kanatani and W. Liu, "3D interpretation of conics and orthogonality", Computer Vision, Graphics and Image Processing, vol. 58, no. 58, pp. 286-301, 1993.

[2] F.L. Bookstein, "Fitting conic sections to scattered data", Computer Graphics and Image Processing, vol. 9, pp. 5671, 1979.

[3] S. Ma, "Conics-based stereo, motion estimation, and pose determination", International Journal of Computer Vision, vol. 10, no. 1, pp. 7-25, 1993.

[4] R. Safaee-Rad, I. Tchoukanov, B. Benhabib, and K.C. Smith, "3D pose estimation from a quadratic curved feature in two perspective views", in Proceedings of the 11th International Conference on Pattern Recognition, The Hague, Netherland, 1992, pp. 341-344.

[5] J.L. Mundy and A. Zisserman, Eds., Geometric Invariance in Computer Vision, MIT Press, Cambridge, Massachusetts, USA, 1992.

[6] A. Zisserman, C. Marinos, D.A. Forsyth, J.L. Mundy, and C.A. Rotwell, "Relative motion and pose from invariants", in Proceedings of the British Machine Vision Conference, Oxford, England, September 1990, pp. 7-12.

[7] M. Ferri, F. Mangili, and G. Viano, "Projective pose estimation of linear and quadratic primitives in monocular computer vision", Computer Vision, Graphics and Image Processing, vol. 58, no. 1, pp. 66-84, July 1993.

[8] S.J. Maybank, "The projection of two non-coplanar conics", in Geometric Invariance in Computer Vision, J. Mundy and A. Zisserman, Eds., pp. 105-119. MIT Press, 1992.

[9] L. Quan, "Algebraic and geometric invariant of a pair of non-coplanar conics in space", Journal of Mathematical Imaging and Vision, 1995, to appear.

[10] L. Quan, "Invariant of a pair of non-coplanar conics in space: Definition, geometric interpretation and computation", in Proceedings of the 5th International Conference on Computer Vision, Cambridge, Massachusetts, USA, 1995, pp. 926-931, to appear.

[11] S.D. Ma, S.H. Si, and Z.Y. Chen, "Quadric curve based stereo", in Proceedings of the 11th International Conference on Pattern Recognition, The Hague, Netherland, 1992. pp. $1-4$.

[12] O. Faugeras, Three-Dimensional Computer Vision - A Geometric Viewpoint, Artificial intelligence. M.I.T. Press, Cambridge, MA, 1993.

[13] R.I. Hartley, "Projective reconstruction and invariants from multiple images", IEEE Transactions on Pattern Analysis and Machine Intelligence, vol. 16, no. 10, pp. 1036-1041, October 1994.

[14] Q. T. Luong and T. Vieville, "Canonic representations for the geometries of multiple projective views", in Proceedings of the 3rd European Conference on Computer Vision, Stockholm, Sweden, May 1994, pp. 589-599.

[15] H.C. Longuet-Higgins and Prasdny, "The interpretation of a moving retinal image", in Proceedings of the Royal Society of London, 1980, pp. 385-397.

[16] R.Y. Tsai and T.S. Huang, "Uniqueness and estimation of 3D motion parameters of rigid bodies with curved surfaces", in IEEE Transactions on Pattern Analysis and Machine Intelligence, vol. 6, pp. 13-27. IEEE Computer Society Press, 1984.

[17] O.D. Faugeras, Q.T. Luong, and S.J. Maybank, "Camera Self-Calibration: Theory and Experiments", in Proceedings of the 2nd European Conference on Computer Vision, Santa Margherita Ligure, Italy, G. Sandini, Ed. May 1992, pp. 321-334, Springer-Verlag.

[18] Q.T. Luong, Matrice Fondamentale et Autocalibration en Vision par Ordinateur, Thèse de doctorat, Université de Paris-Sud, Orsay, France, December 1992.

[19] R. Hartley, R. Gupta, and T. Chang, "Stereo from uncalibrated cameras", in Proceedings of the Conference on Computer Vision and Pattern Recognition, Urbana-Champaign, Illinois, USA, 1992, pp. 761-764.

[20] J.G. Semple and G.T. Kneebone, Algebraic Projective Geometry, Oxford Science Publication, 1952.

[21] P. Lancaster, Lambda-matrices and Vibrating Systems, Pergamon Press, 1966.

[22] G.H. Golub and C.F. Van Loan, Matrix Computation, The Johns Hopkins University Press, Baltimore, 1989. 
TABLE VII

THE RECONSTRUCTION RESUlts of THE THREE CONICS OF THE BREAKFast images.

\begin{tabular}{|c|c|c|c|c|}
\hline & $\Delta$ & $\Theta$ & $\sigma_{3} / \sigma_{2}$ & Plane pair $\left(n^{T},-d\right)$ \\
\hline bowl & -0.001 & 48.6 & 0.008 & $\begin{array}{c}\mathbf{0 . 9 1 8 1}, \mathbf{- 0 . 0 5 0 9 2 ,}-\mathbf{- 0 . 3 9 3 0 ,} \mathbf{1 1 . 8 5}) \\
(-0.1349,-0.9518,0.2753,8.492)\end{array}$ \\
\hline dish inside & -0.0001 & 274.2 & 0.05 & $\begin{array}{c}\mathbf{0 . 9 2 1 0},-\mathbf{0 . 0 9 3 0 7 ,}-\mathbf{- 0 . 3 7 8 2 , 6 . 0 9 3 )} \\
(-0.1248,-0.9425,0.3100,7.172)\end{array}$ \\
\hline dish outside & -0.0005 & 230.2 & 0.03 & $\begin{array}{c}(\mathbf{0 . 9 1 3 1}, \mathbf{- 0 . 0 7 8 4 3 ,}-\mathbf{- 0 . 4 0 0 1 ,} \mathbf{6 . 8 8 0}) \\
(-0.1430,-0.9431,0.3002,7.377)\end{array}$ \\
\hline
\end{tabular}

[23] F. Uhlig, "A canonical form for a pair of real symmetric matrices that generate a non singular pencil", Linear Algebra and Its Applications, vol. 14, pp. 189-209, 1976.

[24] D. Forsyth, J.L. Mundy, A. Zisserman, C. Coelho, A. Heller, and C. Rothwell, "Invariant descriptors for 3D object recognition and pose", IfEe Transactions on Pattern Analysis and Machine Intelligence, vol. 13, no. 10, pp. 971-991, October 1991.

[25] L. Quan, P. Gros, and R. Mohr, "Invariants of a pair of conics revisited", Image and Vision Computing, vol. 10, no. 5, pp. 319-323, June 1992.

[26] L. Robert and O. Faugeras, "Relative 3D positionning and 3D convex hull computation from a weakly calibrated stereo pair", in Proceedings of the 4 th International Conference on Computer Vision, Berlin, Germany, May 1993, pp. $540-544$.

[27] R. I. Hartley, "Cheirality invariants", in Proceedings of DARPA Image Understanding Workshop, 1993, pp. 745753.

[28] K. Kanatani, Geometric Computation for Machine Vision, Oxford Science Publications, Oxford, 1993.

[29] L. Quan and R. Mohr, "Using geometric model guided active contour method for traffic sign detection", in Proceedings of IAPR International Workshop on Machine Vision Application, Tokyo, Japan, 1990.

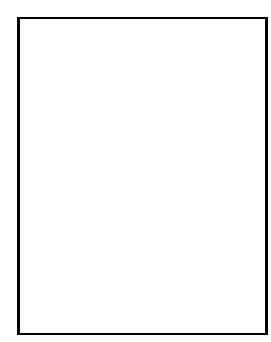

Long Quan was born in Taiyuan, China, on Juin 14, 1964. He received the B.Sc. degree in Telecommunication from the Northern Jiao-Tong Univerity, Beijing, China in 1984, the M.Sc. degree in Computer Science from the University of Nancy I in 1986, and Ph.D. degree in Computer Science from INPL (Institut National Polytechnique de Lorraine), Nancy, France, in 1989.

He joined LIFIA (Laboratoire d'Informatique Fondamentale et d'Intelligence Artificielle), Grenoble, France, as Research Scientist of CNRS (Centre National de la Recherche Scientifique) in 1990. His main research interests are currently on 3D reconstruction, applications of invariant theory and projective geometry for computer vision. 